\title{
Fast Faraday cup to measure neutralized drift compression in intense ion charge bunches
}

\author{
A. B. Sefkow, R. C. Davidson, P. C. Efthimion, and E. P. Gilson \\ Plasma Physics Laboratory, Princeton University, Princeton, New Jersey 08543, USA
}

\author{
S. S. Yu, P. K. Roy, F. M. Bieniosek, J. E. Coleman, S. Eylon, W. G. Greenway, E. Henestroza, J. W. Kwan, \\ D. L. Vanecek, and W. L. Waldron \\ Lawrence Berkeley National Laboratory, Berkeley, California 94720, USA \\ D. R. Welch \\ Voss Scientific, Albuquerque, New Mexico 87108, USA \\ (Received 20 March 2006; published 18 May 2006)
}

\begin{abstract}
Heavy ion drivers for heavy ion fusion and high energy density physics applications use space-chargedominated ion beams which must undergo longitudinal bunch compression in order to meet the requisite beam intensities desired at the target. The Neutralized Drift Compression Experiment-1A (NDCX-1A) at Lawrence Berkeley National Laboratory is used to determine the effective limits of neutralized drift compression, which occurs due to an imposed longitudinal velocity tilt on the drifting beam and subsequent neutralization of the beam's space charge with background plasma. The accurate and temporally resolved measurement of the ion beam's current and pulse length, which has been longitudinally compressed to a few nanoseconds duration at its focal plane, is a critical diagnostic. This paper describes the design and experimental results for a fast and accurate ion beam probe, which reliably measures the absolute beam current in the presence of high density plasma at the focal plane as a function of time. A particle-in-cell code has been used to model the propagation of the intense ion beam and to design the diagnostic probe.
\end{abstract}

PACS numbers: 52.70.- m, 52.65.Rr, 52.59.Sa, 52.40.Mj

\section{INTRODUCTION}

One of the more significant challenges in developing heavy ion drivers is found in the final transport section leading to the target, where ion beam compression in space and time is required in order to achieve conditions necessary for heavy ion fusion and high energy density physics applications. In a heavy ion driver, intense beams of ions [1-3] are transported through a final-focus magnet system to the target chamber, where they must transversely focus onto the target with a final diameter of a few millimeters or less [4,5]. In addition, the ion beams need to be focused longitudinally in order to achieve large amounts of beam current in pulses with durations less than 10 nanoseconds. By greatly compressing the current densities of intense ion charge bunches over short distances, heavy ion drivers can deliver more intensity to the target per unit length of accelerator, allowing for the construction of more compact and cost-effective drivers.

In order to focus an intense ion beam to such a small spot size in a short distance, the majority of the ion beam's space charge must be neutralized during the final transport stage [6,7]. A high density plasma, positioned between the final-focus magnets and the target chamber, provides an electron population which neutralizes the beam's space charge and allows the intense beams to be focused beyond the regular space-charge limit [8].

The Heavy Ion Fusion Science Virtual National Laboratory constructed the neutralized transport experi- ment (NTX) at the Lawrence Berkeley National Laboratory in order to study, on a reduced scale, the physics of the final-focus and neutralized transport of space-charge-dominated beams [9]. The experiment was equipped with an injector which generated a low-emittance and variable-perveance $K^{+}$ion beam capable of up to $400 \mathrm{keV}$ of directed energy. The ion beam was passed through a magnetic-focusing section in order to study the magnet tuning physics and its effects on the phase-space evolution of the beam. At the end of the magnetic-focusing section, the final magnet set was used to give the beam a convergent angle just before the beam entered the drift region, which contained the neutralizing background plasma. The plasma electrons provided sufficient neutralization of the beam's space charge such that the proper convergent angle, applied by the final-focus magnet, allowed the beam to transversely focus to a final spot radius of about 1 millimeter at the focal plane, corresponding to a transverse compression in number density of about $10^{3}$. A more detailed description of the beam line system [9], results of the transverse compression physics [10], comparisons to particle-in-cell (PIC) simulations [11], and other important experimental topics addressed by the NTX experiments [12] were recently published.

Successfully focusing an ion beam in the transverse direction is only part of the challenge. In order to achieve the requirement that the pulse length be less than 10 nanoseconds when the focal plane is reached, each ion beam 


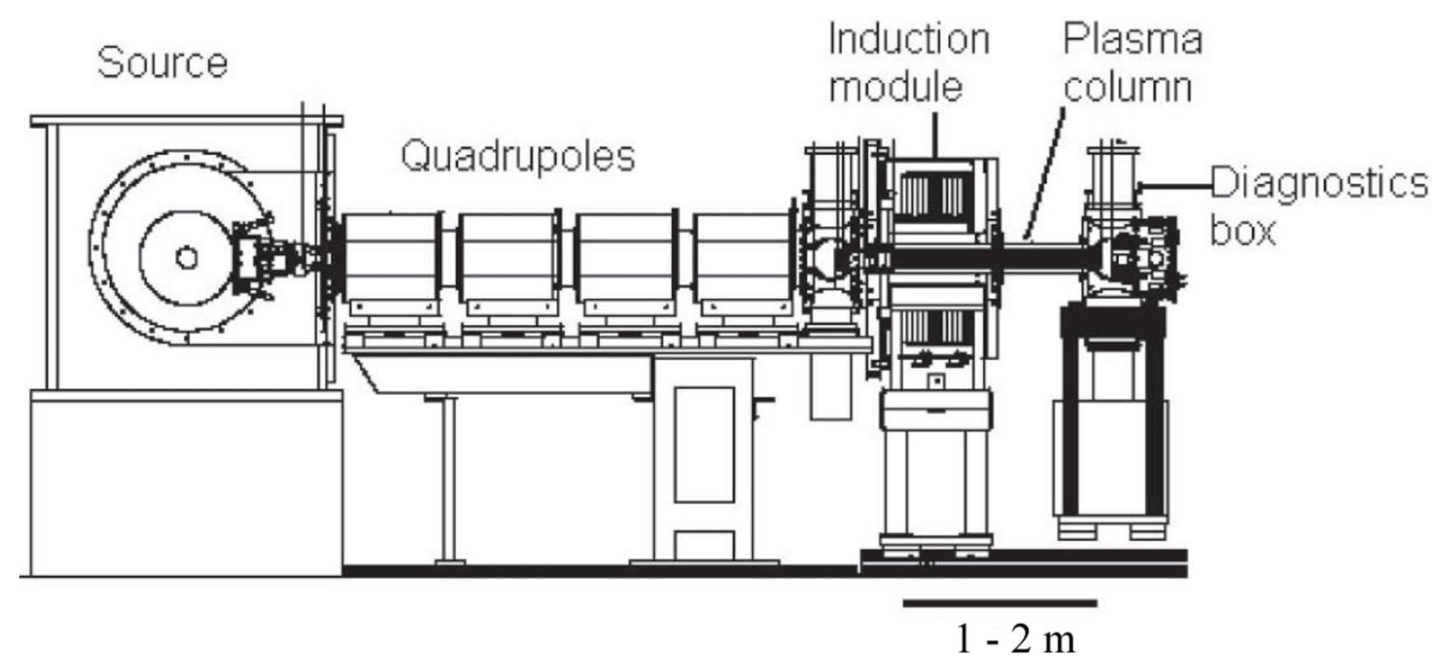

FIG. 1. Schematic of the NDCX-1A experimental setup.

pulse must also be compressed longitudinally, along the direction of propagation. In order to meet the requisite current densities on target, there is a need to determine the physical and technological limits of such longitudinal focusing, achieved by applying a time-dependent velocity tilt to the beam and subsequently allowing it to drift through a background plasma, thereby neutralizing the beam's space charge as the beam's pulse length compresses longitudinally [13]. This concept is referred to as "neutralized drift compression," and the upgrade of the NTX facility is called the Neutralized Drift Compression Experiment-1A (NDCX-1A). Longitudinal compression of space-charge-dominated ion beams without the aid of background neutralizing plasma has also been studied $[14,15]$, but the associated longitudinal compression was significantly reduced relative to the neutralized case, due to the effects of ion beam space charge.

A schematic of the NDCX-1A experimental setup is shown in Fig. 1. On NDCX-1A, the primary addition to NTX is the induction module, which is a linear induction accelerator [16] with one acceleration gap that applies the required axial velocity tilt to the beam by means of a timedependent voltage pulse. The time-dependent voltage pulse has two extremum, with a change in polarity at the halfway point, such that the first half of the beam pulse (the "head") experiences a net decelerating electric force, and the second half of the beam pulse (the "tail") experiences a net accelerating electric force. Thus, the beam pulse acquires an initial head-to-tail velocity tilt immediately before it drifts through the region containing background plasma. After the beam has drifted through the plasma column, a focal plane in current is reached. Since the current density, and therefore the relative power, of the charge bunch grows in the intermediate region of the applied tilt, where the decelerated head meets the accelerated tail, while the beam drifts through a background plasma, the phrase "neutralized drift compression" explicitly refers to this process. The two main goals of the NDCX-1A campaign are to determine the limits of how much current density compression can be achieved experimentally and how accurately those compression factors can be measured. Simulations using the LSP PIC code predict a pulse width of a few nanoseconds with up to about one $A / \mathrm{cm}^{2}$ of current density at peak compression for initial experiments [17]. An accurate measurement of the total current of a longitudinally compressed intense ion charge bunch is important in order to determine the compression factors achieved in a variety of experimental conditions and make comparisons to theory and simulations.

The ion beam probe that was designed to measure the beam current $I_{b}(t)$, while operating in the presence of high density plasma in the NDCX-1A beam line, is a modified Faraday cup. Figure 2 shows a schematic of a standard Faraday cup. Generically, a Faraday cup consists of a negatively biased, electron-repelling ring located "upstream" of a positively biased collector cup and enclosed in a grounded housing such that plasma particles and secondary electrons in the system are controlled. A stan-

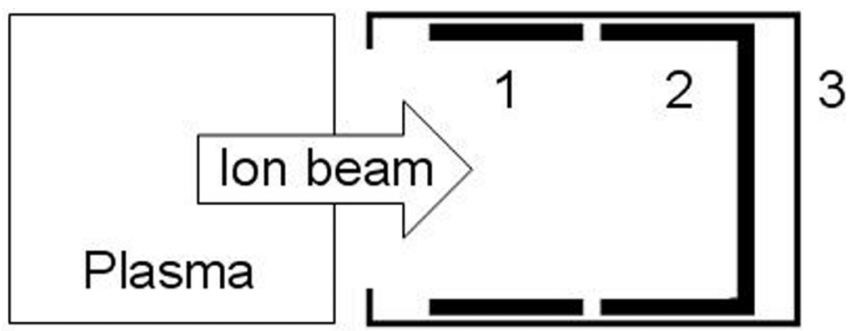

FIG. 2. Standard Faraday cup diagnostic for $I_{b}(t)$ : (1) negatively biased electron-repelling ring; (2) positively biased collector cup; and (3) grounded enclosure. 
dard Faraday cup design that admits the compressed ion beam in its entirety is inadequate for the purposes of the NDCX-1A experiments because of the complex plasma and secondary electron behavior within such a probe. For instance, if high density plasma is allowed to enter the probe, it can shield the applied potentials which were meant to reject all particles from measurement except the beam ions, allowing plasma electrons (and ions) to penetrate the probe and be detected, thereby reducing (and increasing) the corresponding signal measured at the collector. Thus, if plasma particles are not controlled, the signal due only to the beam ions cannot reliably be determined by such a measurement. Ideally, an experimenter would prefer that the collector only measure a signal proportional to the ion beam current. Therefore, a fast Faraday cup diagnostic for measuring the absolute beam current in the presence of plasma at the focal plane as a function of time has been designed, fabricated, and incorporated into the NDCX-1A beam line [17]. The fast Faraday cup diagnostic has corroborated optical measurements made by a fast phototube arrangement [18] which measures light intensity created by beam bombardment on a scintillator. However, the limitation of such an optical measurement is that it cannot measure the actual ion beam current, whereas the fast Faraday cup diagnostic can.

The simulations used to design the fast Faraday cup for operation within high density plasma are described in Sec. II. In Sec. III, a discussion of the probe's capabilities and limitations is presented, as well as experimental results of neutralized drift compression that were measured using the fast ion beam diagnostic.

\section{FAST FARADAY CUP SIMULATIONS AND DESIGN}

The successful characterization of neutralized drift compression in the NDCX-1A device requires accurate measurements of the compressed ion beam current, $I_{b}(t)$, current density, $J_{b}(x, y, z, t)$, and pulse width at the focal plane near the end of the plasma region. Several probe designs, based on numerical simulations, were tested to measure these parameters of the beam in the presence of a high density plasma. The use of the LSP PIC code is one method for simulating an ion beam pulse propagating through a background plasma. LSP [19,20] is an advanced 3D electromagnetic PIC code, available commercially and designed for complex, large-scale plasma simulations which can be run in multiple coordinate systems and geometries. The LSP code has been utilized in order to realistically predict the evolution of the ion beam as it acquires a velocity tilt and drifts through the plasma to the focal plane. The code has also been employed to determine the requirements necessary for an accurate measurement of the beam's current using a probe which resides in the plasma environment.
There are five key requirements in order to accurately measure the true $I_{b}(t)$ or $J_{b}(x, y, z, t)$ in NDCX-1A. These are: (1) plasma ions and beam ions must not shield the negatively biased electron-repelling ring; (2) comoving plasma electrons that neutralize the beam's potential must be completely rejected from the probe; (3) beam ions must not be allowed to strike the electron-repelling ring and create secondary electrons which would foul the measurement at the collector; (4) secondary electrons created by ion bombardment on the collecting surface must be trapped, otherwise artificially high signals would be measured at the collector; and (5) large beam current densities must be measured at the collector with subnanosecond time resolution.

In order for the diagnostic to operate properly in a plasma environment, LSP simulations indicate that the standard Faraday cup design should be modified such that it includes a grounded frontplate containing plasma Debye-length-size holes, replaces the electron-repelling ring with an electron-repelling plate containing slightly larger holes, and replaces the collector cup with a collector plate, as shown in Fig. 3. The aligned holes of the frontplate and electron-repelling plate (also called the backplate) provide a geometric shadow to the probe, thereby allowing only a fraction of the compressed beam pulse to enter the diagnostic and simplifying the control of the five important processes listed above. The frontplate and backplate are similar in design and purpose to grids normally found in a gridded-energy analyzer [21]. Thus, the modified Faraday cup could also be described as a type of gridded-energy

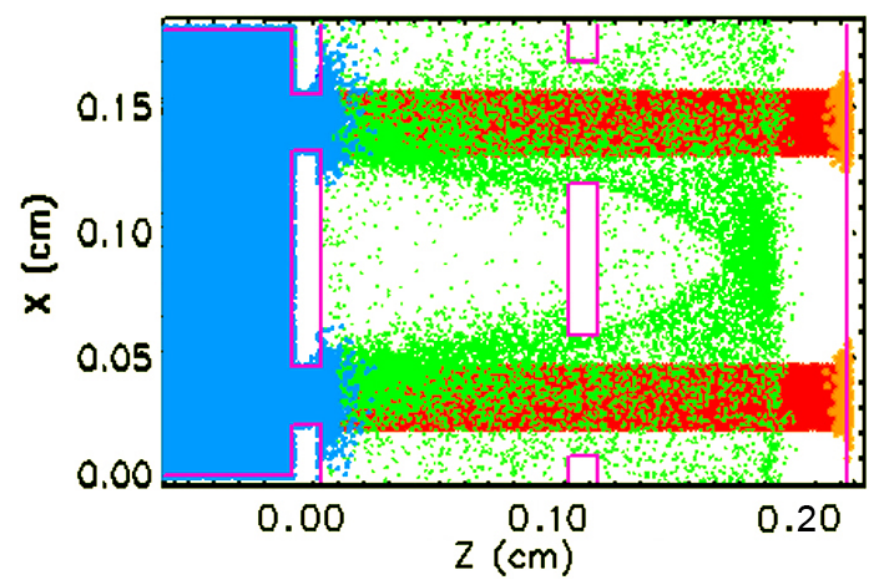

FIG. 3. (Color) Particle plot from an LSP simulation of the pinhole Faraday cup diagnostic for $I_{b}(t)$ (only a fraction of the total number of particles are plotted for clarity reasons): (red) $\mathrm{K}^{+}$ion beam particles; (blue) plasma electron particles; (green) plasma ion particles; (orange) secondary electron particles. The grounded frontplate $(z=0 \mathrm{~mm})$, the negatively biased electronrepelling backplate $(z=1 \mathrm{~mm})$, and the positively biased collector cup $(z=2 \mathrm{~mm})$ are shown. Secondary electrons created by plasma ion bombardment on the backplate are not shown for clarity reasons. 
analyzer which measures beam current rather than directed energy.

The LSP simulations modeled five species of particles: (1) the plasma electrons, (2) the plasma ions $\left(\mathrm{Al}^{+}\right),(3)$ the beam ions $\left(\mathrm{K}^{+}\right)$, (4) secondary electrons created on the frontplate and collector by ion beam bombardment, and (5) secondary electrons created on the frontplate and backplate by plasma ion bombardment (due to the design requirements outlined earlier, beam ions did not strike the backplate and plasma ions did not strike the collector). In the LSP simulations, all species were treated as kinetic, explicit, and energy-conserving particles, the electrostatic field equations were solved (meaning the $\partial \mathbf{E} / \partial t$ terms and $\partial \mathbf{B} / \partial t$ terms were ignored), and a cloud-in-cell interpolation scheme was used in order to reduce particle noise [22]. In addition, a stimulated secondary electron emission package (included in LSP) was used in order to model the ejection of electrons from conducting surfaces when beam ion or plasma ion particles struck any of the conducting surfaces of the simulation (the frontplate, backplate, and collector).

The major difference between the standard Faraday cup design and the new "pinhole" design is found in the nature of the negatively biased, electron-repelling backplate. In the standard Faraday cup design, shown in Fig. 2, the electron-repelling ring only exists out at some large radius (greater than the ion beam's radius) and does not adequately remove all of the comoving electrons from the beam's potential. Instead, shielding occurs by the various charged particle populations and the potential found closer to the axis is no longer adequate to remove all of the electrons from the ion beam charge bunch. Therefore, the signal detected at the collector would not be proportional to the beam current alone, but would be reduced by the amount of plasma electron current detected at the collector. Also, the plasma ions are accelerated into the electronrepelling ring by the large, negative bias applied to that ring. Those plasma ions can eject secondary electrons and the LSP simulations show that such electrons can become greatly accelerated by the ring and also be detected, further complicating the signal at the collector.

In the pinhole Faraday cup design, shown in Fig. 3, a grounded frontplate with plasma Debye-length-size holes $\left(n_{p} \sim 10^{10} \mathrm{~cm}^{-3}, \mathrm{kT}_{p} \sim 3 \mathrm{eV}\right)$ admits only a small fraction of the beam, while also limiting the effective probe entry locations for the plasma ions and electrons. The frontplate is grounded so as to minimize the perturbation to the surrounding plasma environment. Since the entrance holes are on the order of the plasma Debye length, the ion beamlets (created by the shadow of the frontplate) are each stripped of their neutralizing, comoving plasma electrons because each frontplate hole has some corresponding negatively biased, electron-repelling backplate in close proximity. The backplate with slightly larger holes (to accommodate diverging beam ions and avoid the creation of energetic secondary electrons by ion beam bombardment on the backplate) replaces the electron-repelling ring of the standard Faraday cup and is effective at resisting the introduction of plasma electrons into the diagnostic. Thus, plasma electrons (as well as secondary electrons created on the frontplate by ion beam bombardment) are secluded outside of the probe due to the electric field created between the frontplate and backplate. Plasma ions are, in fact, accelerated into the probe by such an electric field, however they are repelled from detection by an applied positive bias on the collector plate which creates a stronger, reversed electric field between the backplate and the collector. The positive bias on the collector also serves to trap secondary electrons, created by energetic heavy ion beam bombardment, on the collector plate so that the measured signal is proportional to the ion beam current (and not artificially high due to secondary electron loss from the detector). The energetic ion beamlets pass into the diagnostic largely unaffected by the biases, since the applied potentials on the backplate and collector are about $5 \times$ $10^{-4}$ lower than the directed energy of the ion beam pulse. The trajectories of the beam ions are expected to be unperturbed by sheath effects.

According to LSP simulations, since the frontplate holes are comparable in size to the plasma Debye length $\left(n_{p} \sim\right.$ $10^{10} \mathrm{~cm}^{-3}, \mathrm{kT}_{p} \sim 3 \mathrm{eV}$ ), and because the plasma ions are modeled with only thermal and no directed energy (plasmas with axial drift momentum can be repelled using higher biases), the densities associated with the plasma ions penetrating into the diagnostic are expected to be two to three orders of magnitude lower $\left(\sim 10^{7}-\right.$ $10^{8} \mathrm{~cm}^{-3}$ ) than the bulk plasma density outside of the probe. Therefore, any image-current effects and detection of secondary electron currents created by (low energy) plasma ion bombardment on the backplate are expected to be negligible ( $<10^{-3}$ of the ion beam signal). Thus, all three possible sources of secondary electron current in the diagnostic are controlled (ion beam and plasma ion bombardment on the backplate, as well as ion beam bombardment on the collector), and their negative side effects on the measurement process are mitigated. The fourth source of secondaries, the ion beam bombardment on the frontplate, was simulated as well, however those electrons are completely rejected from the probe by the same mechanism which rejects the plasma electrons.

The plasma ions, the secondary electrons created on the backplate by plasma ion bombardment, and the secondary electrons created on the collector by ion beam bombardment do not significantly shield the potentials on the backplate and collector, due to the close (1 millimeter) spacings between the three plates (the associated Debye lengths for these species are $>1$ millimeter due to their low densities). The 1 millimeter gaps were chosen to reduce the bias-shielding capability of the beam ions and to minimize induced image-current effects at the collector. Therefore, 
TABLE I. Pinhole Faraday cup specifications.

\begin{tabular}{lccc}
\hline \hline Plate & $r_{\text {hole }}$ & Hole-to-hole & Bias \\
\hline Front & $114 \mu \mathrm{m}$ & $1016 \mu \mathrm{m}$ & Grounded \\
Back & $241 \mu \mathrm{m}$ & $1016 \mu \mathrm{m}$ & $\begin{array}{c}-150 \mathrm{~V} \\
+50 \mathrm{~V}\end{array}$ \\
Collector & & & \\
\hline \hline
\end{tabular}

the entire beam (less some transmission factor due to the geometric shadow cast by the frontplate) will be admitted into the probe and the collector will accurately measure the total beam current, $I_{b}(t)$, before the beam has sufficient time to decompress due to space-charge forces. This configuration has been shown in LSP simulations to be ideal for controlling the two most important electron populations: if all comoving plasma electrons were not rejected from detection, then the signal on the collector plate would underestimate $I_{b}(t)$; if all secondary electrons created on the collector by ion beam bombardment were not trapped, then the signal on the collector plate would overestimate $I_{b}(t)$.

The probe used in the initial experiments [23] on NDCX-1A consists of a frontplate with hole radii approximately a plasma Debye length in size $\left(n_{p} \sim 10^{10} \mathrm{~cm}^{-3}\right.$, $\mathrm{kT}_{p} \sim 3 \mathrm{eV}$ ), followed 1 millimeter "downstream" by a backplate with larger hole radii, and a collector plate another millimeter downstream of the backplate. Table I shows the hole radius, the horizontal hole-to-hole separation, and the applied bias for each of the plates. The vertical hole-to-hole separation is $\sqrt{3} / 2$ times the horizontal separation, and each row of holes is staggered with respect to the adjacent rows, giving a hexagonal arrangement. The

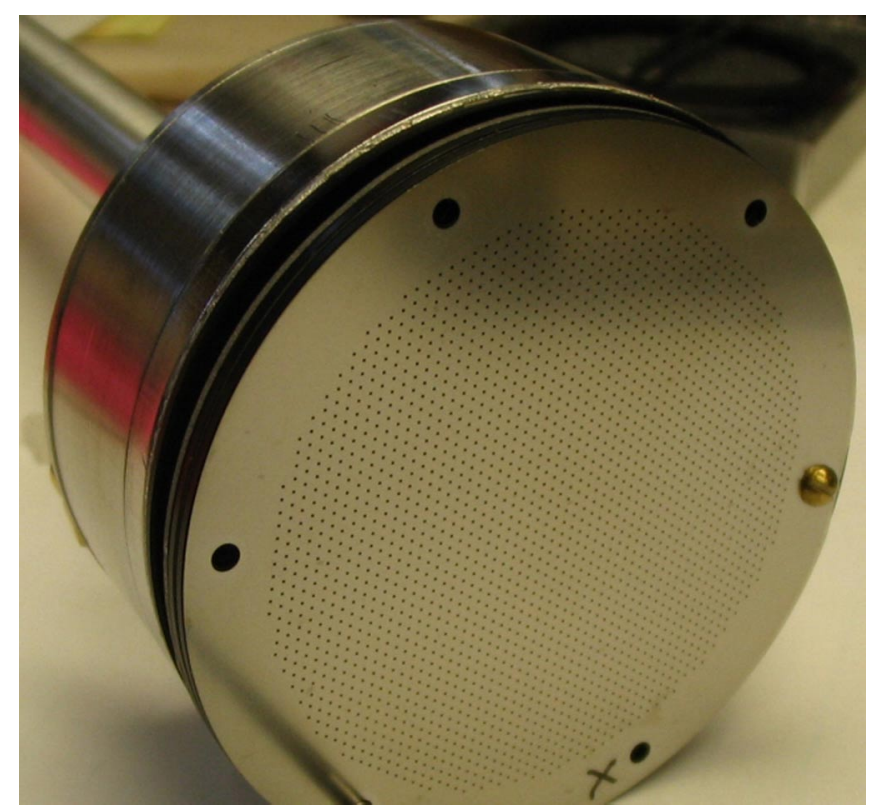

FIG. 4. (Color) Photograph of the pinhole Faraday cup diagnostic before installation on NDCX-1A. frontplate and backplate are each $102 \mu \mathrm{m}$ thick ( $\left.<2 r_{\text {hole }}\right)$, made of stainless steel, and contain over 2000 holes. Both of the hole plates were fabricated by a commercial photoetching process. Because of the geometrical shadow cast by the holes in the frontplate, the signal measured at the collector must be multiplied by a transmission factor in order to determine the total beam current. The probe biases were chosen such that the diagnostic would operate properly under the harshest conditions expected at the focal plane for present experiments $\left(n_{\text {plasma }}^{\text {peak }}=10^{11} \mathrm{~cm}^{-3}\right.$, $n_{\text {beam }}^{\text {peak }}=10^{10} \mathrm{~cm}^{-3}$ ).

A photograph of the fast Faraday cup is shown in Fig. 4. The signal measured at the collector plate is expected to be very close to the true ion beam current. Error levels associated with measuring only the ion beam particles are expected to be $<1 \%$. Signal fidelity, instrumentation response, and various sources of noise are expected to be the chief sources of error for measurements in experiments on the NDCX-1A device.

\section{EXPERIMENTAL RESULTS}

Measurements made with the pinhole Faraday cup show that, when only plasma is present, the collector plate draws no current and the backplate draws about $0.2 \mathrm{~mA}$ of plasma ion current, in agreement with LSP simulations assuming plasma density $n_{p} \sim 10^{10} \mathrm{~cm}^{-3}$. For beam measurements, the injector produces a 6 microsecond-long, $299 \mathrm{keV} \mathrm{K}^{+}$ beam of approximately 2 centimeter radius which is transported in the $+\hat{z}$ direction through the magnetic-focusing and plasma drift sections. Its current is then measured by the probe in the presence of background plasma.

In order to calibrate the new diagnostic, measurements of beam current were taken with the fast Faraday cup and compared to previous measurements using the standard Faraday cup without background plasma present in the

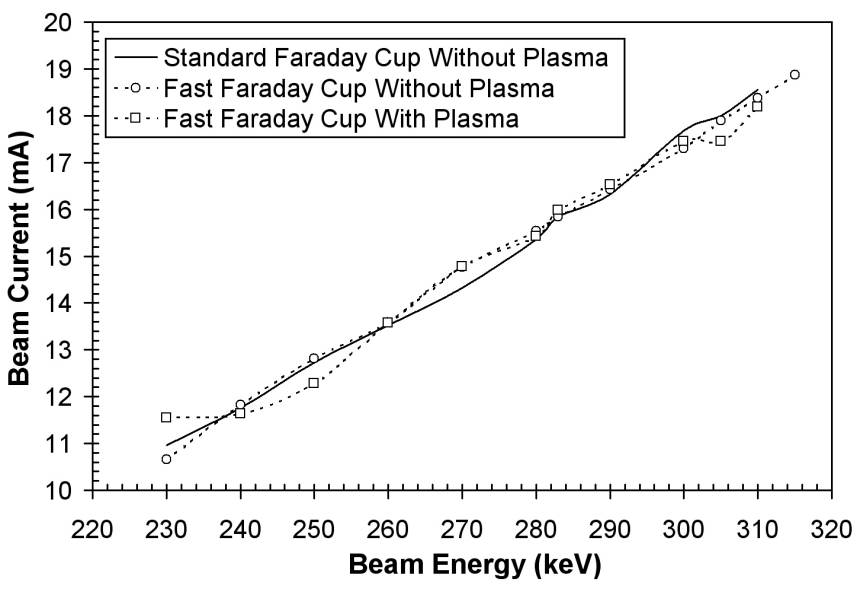

FIG. 5. Uncompressed ion beam current, $I_{b}$, as a function of beam energy: (solid line) standard Faraday cup without plasma; (dotted line, circles) fast Faraday cup without plasma; (dotted line, squares) fast Faraday cup with plasma. 
experiment. Figure 5 shows very good agreement between the fast and standard Faraday cups when the (uncompressed) beam current is measured without a background plasma over a wide range of beam energies. Note that this is the only regime where the standard Faraday cup gives accurate measurements (it cannot accurately measure the beam current in the presence of plasma, which is required for significant compression). The measurements were repeated with the fast Faraday cup in the presence of background plasma in order to test whether the diagnostic operates properly in such an environment. Figure 5 clearly shows that the fast Faraday cup measures the true beam current magnitude in the presence of background plasma. The calibration of the fast Faraday cup is satisfactory since the error has been measured to be $<3 \%$ over most of the range of comparison to the standard Faraday cup measurements (especially at higher energies where most compression data is taken).

In order to compress the ion beam in the longitudinal direction, the time-dependent voltage imposes a velocity tilt on the ion beam. The beam drifts through a plasma column until a focal plane in current density is created between 1 and 2 meters downstream of the gap (the length of the plasma column can be changed from 1 to 2 meters in the experiment). Neutralization of the beam's space charge by background plasma is required in order to compress the beam to current densities of interest for heavy ion fusion and high energy density physics applications. The exact location of the focal plane of the compressing beam is determined by the slope of the voltage waveform applied to the induction module, as well as by the velocity of the injected ion beam. In these experiments, the induction module's timing and voltage swing act on the middle 2

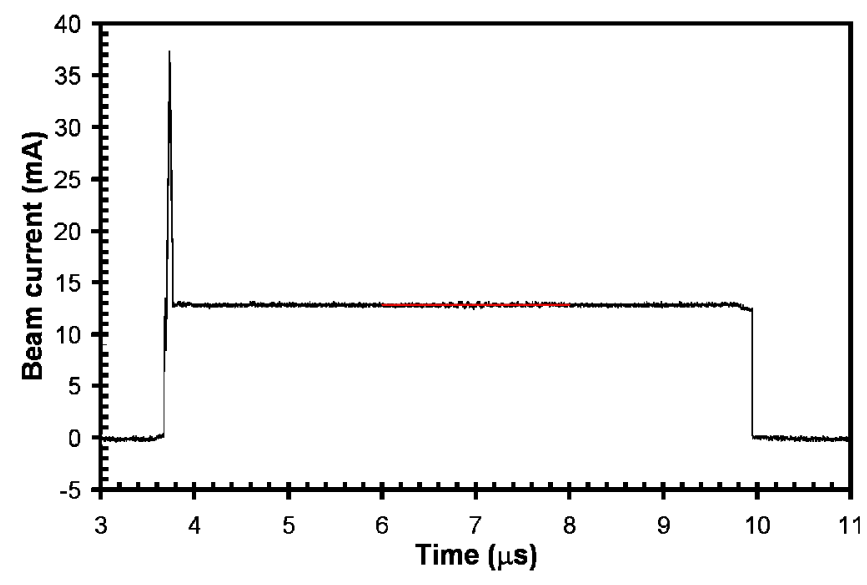

FIG. 6. (Color) Uncompressed ion beam current, $I_{b}(t)$. Shown is the average of 11 shots (without an applied velocity tilt) measured with the fast Faraday cup. The velocity tilt is applied in the middle 2 microseconds of the ion beam pulse. Time is measured from the trigger of the voltage pulse on the source injector so that the rise of the signal on the collector also measures the transit time of the beam particles from the injector to the diagnostic. microseconds of the 6 microsecond-long beam. The fast Faraday cup is located at the far end of the plasma region such that the longitudinal focal plane coincides with the plane of the collector plate. Figure 6 shows the ion beam current $I_{b}(t)$ measured by the collector plate; it illustrates the level of noise latent in the uncompressed beam signal once the data has been averaged over a few shots and has accounted for the transmission factor of the incident beam. The sharp peak measured early in time is a commonly seen feature primarily associated with the pileup of the slower particles in the head of the ion beam pulse, created by the finite amount of rise time in the source voltage. The feature may also be associated with effects such as image current and latent capacitance in the diagnostic.

Measurements of neutralized drift compression by the fast Faraday cup in the initial experiments [23] revealed the need for electronic modifications to ensure faster temporal response by the probe, which must accurately track the beam's fast-rising and fast-falling signal. The probe design discussed up until now was limited in its ability to resolve signals on the nanosecond time scale, due to circuit and construction details, so the fifth key requirement (see Sec. II) to accurately measure the true ion beam current, $I_{b}(t)$, had not yet been satisfied. Thus, a number of electronic modifications for improving the high frequency response were investigated. Some of the modifications adopted were similar to those used in probes that also required nanosecond temporal resolution, but are used for different applications $[24,25]$.

The modifications from the fast Faraday cup diagnostic first described in [23] include the following. First, the collector plate's material was changed from stainless steel to copper and the coaxial cable connection was moved from the circumference of the collector to the center. Both changes lowered the effective impedance the beam signal encountered. Second, a new housing design was employed to increase the distance between the grounded housing and the plates in order to avoid creating a lumped element capacitance, which deteriorates the response time by increasing the time constant. Third, all signal-bearing cables to the three plates were replaced with wellinsulated, shielded wires in order to avoid inducing unwanted high frequency signals. Fourth, the backplate was coupled to the grounded housing by six $50 \mathrm{nF}$ capacitors in parallel, effectively leaving the biased backplate at AC ground to ensure isolation from signals in the $\mathrm{GHz}$ range. Fifth, circumferential delrin spacers between the plates were replaced with small Teflon washers around the support rods of the probe in order to further decrease the amount of capacitance between the plates. The three plates are required to be close together in order to: (1) diminish image-current effects from the ion beam on the collector; (2) achieve the desired electric field strengths to control the various charged particle populations; and (3) ensure that the beam does not have enough time to decompress before 
measurement. However, an important side effect is the presence of some latent capacitance in the system due to the geometrical arrangement of the plates. The effect is believed to be one of the limiting factors of this design for measuring fast signals.

In addition, other considerations were also addressed in the new fast Faraday cup design. Larger, more closely spaced holes reduced the signal noise and increased the statistical sampling of the beam, at the cost of higher biases in the probe to control the plasma and secondary behavior, as well as degraded structural integrity of the frontplate and backplate. Because of the spacings of the holes in the frontplate, the question arises as to whether submillimeterscale fluctuations in current are lost to the frontplate and averaged over. Measurements made while the probe was in slightly different vertical positions showed that there are no submillimeter fluctuations of importance in the experiment.

The role of desorbed gas from the frontplate and collector due to ion beam bombardment was also considered and its effect on the measurement was concluded to be small. The estimated total surface charge density at the focal plane (where the diagnostic resides) for the $\sim 6 \mu$ s of ion charge bunch which is not longitudinally compressed is $\sim 4 \times 10^{-7} \mathrm{C} \mathrm{cm}^{-2}$. Using the estimate that $\sim 3000$ gas molecules are liberated from the collector plate per impacting beam ion [26], and assuming the probe fills with desorbed gas at a flow rate of $\sim 1 \mathrm{~mm} / \mu \mathrm{s}$ [26], the peak gas density is expected to be $<4 \times 10^{15} \mathrm{~cm}^{-3}$. The corresponding gas pressure is $1.5 \times 10^{-4} \mathrm{~atm}$, which is well below the bottom of the Paschen curve $(p \times d=7.62 \times$ $10^{-3} \mathrm{~mm}$ atm), implying that gas breakdown by the applied electric field in the 1 millimeter gap between the backplate and collector is unlikely (the gas density is even lower between the frontplate and backplate because of the transmission factor of the backplate). Also, the mean-free path for beam-gas collisions is estimated to be larger than the probe's length ( 2 millimeters), therefore the probability of desorbed gas ionization by incoming beam ions is small (which would weaken the beam signal at the collector due to the drawn electron current if gas ionization were significant). Lastly, the small fraction of the beam that was longitudinally compressed to higher current density is only a minor addition to the gas density estimation because the compressed pulse is a factor of $<10^{-3}$ shorter in duration than the original $6 \mu$ s-long charge bunch. In support of these estimates, measurements taken with the fast Faraday cup are found to be clear of any detectable gas effects.

In order to test the new fast Faraday cup design, a set of experiments on the NDCX-1A device were made in order to achieve large longitudinal compression with a short temporal pulse width. A $320 \mathrm{keV} \mathrm{K}^{+}$beam with a $2 \mathrm{~cm}$ radius was injected into the NDCX-1A, a $15 \%$ velocity tilt (defined as $\Delta v_{b} / v_{b}^{\text {initial }}$ ) was applied to the beam by the

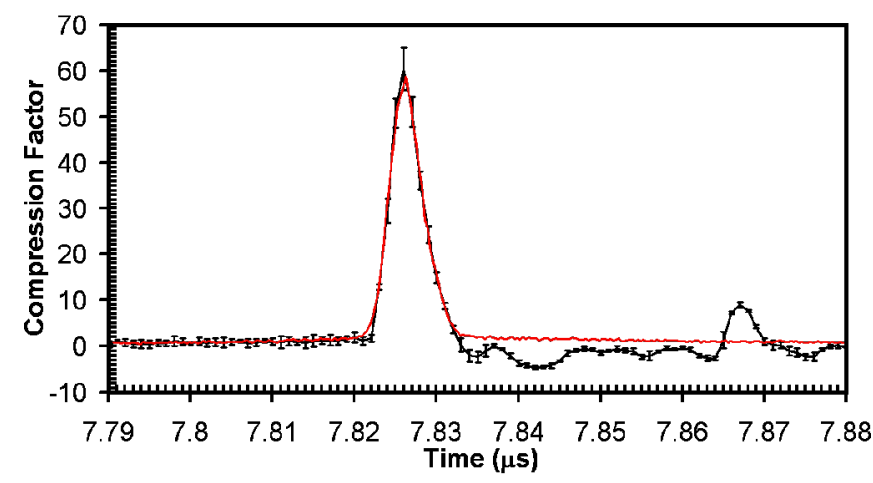

FIG. 7. (Color) Fast Faraday cup measurement of longitudinal current compression: (black) experiment; (red) LSP simulation.

induction module, and the beam drifted through a background plasma of density $\sim 10^{10} \mathrm{~cm}^{-3}$. The experimental results in Fig. 7 show an average of the four best shots measured with the fast Faraday cup at the focal plane, which ranged in compression factors (defined as $\left.I_{b}^{\max } / I_{b}^{\text {initial }}\right)$ from 55 to 65 . The compressed ion beam signal has been normalized to the initial beam current (without an applied tilt) and expanded around the location of peak compression in order to show the error bars. The error bars plotted for the experiment are the 95\% confidence intervals in the mean of the four shots. The measured ringing reflections seen in the experimental signal after the compressed beam pulse are due to impedance mismatch in the $50 \Omega$ circuit.

In order to simulate the experiment, the experimentally measured voltage waveform for the induction module was used in an LSP PIC simulation, the result of which is also plotted in Fig. 7. In the simulation, the ion beam was initialized upstream of the induction module with a $0.2 \mathrm{eV}$ temperature in both the transverse and longitudinal directions and the background plasma density and temperature were $5 \times 10^{10} \mathrm{~cm}^{-3}$ and $3 \mathrm{eV}$, respectively. LSP simulation predicts a compression factor of 60 times the initial current with a full-width, half-maximum pulse width of 4 nanoseconds, in excellent agreement with the fast Faraday cup measurement. Without the neutralizing plasma present, a compression factor of approximately 20 was measured, also in agreement with LSP simulation. It is believed that the shortest achievable pulse widths of the compressed ion beams in these experiments were limited by the voltage waveform applied by the induction module; there exists a slope for the velocity tilt which would ideally compress the beam and deviations from that slope in the experimental waveform are expected to dominate the minimum achievable pulse width in these experiments. If the beam had an ideal slope to its velocity tilt, then chromatic aberration due to longitudinal temperature effects are expected to dominate the minimum achievable pulse width. Other interesting experimental results and simulations pertaining to NDCX-1A, especially as 
they relate to the need for precise control over the slope of the induction module waveform which applies the head-totail velocity tilt to the drifting ion beam, have been published [27]. Lastly, a theoretical model based on a kinetic formalism for simultaneous longitudinal and transverse compression of an intense ion beam has also been published recently [28].

\section{CONCLUSIONS}

A fast Faraday cup diagnostic for measuring the absolute beam current in the presence of plasma at the focal plane as a function of time has been designed, fabricated, and installed on the NDCX-1A device. Experiments have confirmed that the collector only measures a signal proportional to the ion beam current, without the negative influence of the other particle species in the system. The fast Faraday cup diagnostic has corroborated optical measurements made by a scintillator and fast phototube arrangement. However, only the fast Faraday cup diagnostic can measure the actual ion beam current in the presence of plasma.

Future work will include the construction of a current density diagnostic, which will be similar to the $I_{b}(t)$ probe except it will be smaller and movable in all three directions. The $J_{b}(x, y, z, t)$ probe will allow the experimenter to sample the current density at the focal plane as a function of space and time, as well as provide additional insight into the physical and technological limitations of neutralized drift compression. Neutralized drift compression offers the potential of compressing heavy ion beams to very high current densities and, therefore, shorter accelerators could be used as heavy ion drivers for heavy ion fusion and high energy density physics applications, making them more compact and cost effective than previously envisioned.

\section{ACKNOWLEDGMENTS}

This research was supported by the U.S. Department of Energy under the auspices of the Heavy Ion Fusion Science Virtual National Laboratory.

[1] M. Reiser, Theory and Design of Charged Particle Beams (Wiley, New York, 1994).

[2] R. B. Miller, An Introduction to the Physics of Intense Charged Particle Beams (Plenum Press, New York, 1994).

[3] R. C. Davidson and H. Qin, Physics of Intense Charged Particle Beams in High Energy Accelerators (World Scientific, Singapore, 2001).

[4] D. A. Callahan, Fusion Eng. Des. 32-33, 441 (1996).

[5] B. G. Logan and D. A. Callahan, Nucl. Instrum. Methods Phys. Res., Sect. A 415, 468 (1998).

[6] I. D. Kaganovich, G. Shvets, E. Startsev, and R.C. Davidson, Phys. Plasmas 8, 4180 (2001).
[7] D. V. Rose, D. R. Welch, B. V. Oliver, R. E. Clark, W. M. Sharp, and A. Friedman, Nucl. Instrum. Methods Phys. Res., Sect. A 464, 299 (2001).

[8] D. R. Welch, D. V. Rose, B. V. Oliver, and R. E. Clark, Nucl. Instrum. Methods Phys. Res., Sect. A 464, 134 (2001).

[9] E. Henestroza, S. Eylon, P. K. Roy, S. S. Yu, A. Anders, F. M. Bieniosek, W. G. Greenway, B. G. Logan, R. A. MacGill, D. B. Shuman, D. L. Vanecek, W. L. Waldron, W. M. Sharp, T.L. Houck, R.C. Davidson, P.C. Efthimion, E. P. Gilson, A. B. Sefkow, D. R. Welch, D. V. Rose, and C.L. Olson, Phys. Rev. ST Accel. Beams 7, 083501 (2004).

[10] P. K. Roy, S. S. Yu, S. Eylon, E. Henestroza, A. Anders, F. M. Bieniosek, W. G. Greenway, B. G. Logan, W. L. Waldron, D. L. Vanecek, D. R. Welch, D. V. Rose, R. C. Davidson, P. C. Efthimion, E. P. Gilson, A. B. Sefkow, and W. M. Sharp, Phys. Plasmas 11, 2890 (2004).

[11] C. H. Thoma, D. R. Welch, S. S. Yu, E. Henestroza, P. K. Roy, S. Eylon, and E. P. Gilson, Phys. Plasmas 12, 043102 (2005).

[12] P. K. Roy, S.S. Yu, S. Eylon, E. Henestroza, A. Anders, E. P. Gilson, F. M. Bieniosek, W. G. Greenway, B. G. Logan, W. L. Waldron, D. B. Shuman, D. L. Vanecek, D. R. Welch, D. V. Rose, R.C. Davidson, P.C. Efthimion, I. D. Kaganovich, A. B. Sefkow, and W. M. Sharp, Nucl. Instrum. Methods Phys. Res., Sect. A 544, 255 (2005).

[13] D. R. Welch, D. V. Rose, S. S. Yu, J. J. Barnard, and C. L. Olson, Nucl. Instrum. Methods Phys. Res., Sect. A 544, 236 (2005).

[14] H. Qin, R. C. Davidson, J. J. Barnard, and E. P. Lee, Phys. Rev. ST Accel. Beams 7, 104201 (2004).

[15] W. M. Sharp, J. J. Barnard, D. P. Grote, C. M. Celata, and S. S. Yu, Nucl. Instrum. Methods Phys. Res., Sect. A 544, 398 (2005).

[16] N. Christofilos, R. Hester, W. Lamb, D. Reagan, W. Sherwood, and R. Wright, Rev. Sci. Instrum. 35, 886 (1964).

[17] A. B. Sefkow, R.C. Davidson, P.C. Efthimion, E.P. Gilson, S. S. Yu, P. K. Roy, S. Eylon, F. M. Bieniosek, E. Henestroza, J.E. Coleman, W. L. Waldron, W. G. Greenway, D. L. Vanecek, and D. R. Welch, Bull. Am. Phys. Soc. 50, 49 (2005), http://meetings.aps.org/Meeting/ DPP05/Event/34629.

[18] F. M. Bieniosek, S. Eylon, A. Faltens, A. Friedman, J. W. Kwan, M. A. Leitner, A. W. Molvik, L. Prost, P. K. Roy, P. A. Seidel, and G. Westenskow, Nucl. Instrum. Methods Phys. Res., Sect. A 544, 268 (2005).

[19] LSP is a software product of ATK Mission Research, Albuquerque, NM 87110.

[20] T. P. Hughes, S.S. Yu, and R.E. Clark, Phys. Rev. ST Accel. Beams 2, 110401 (1999).

[21] I.H. Hutchinson, Principles of Plasma Diagnostics (Cambridge University Press, Cambridge, England, 2002).

[22] C. K. Birdsall and A. B. Langdon, Plasma Physics via Computer Simulation (McGraw-Hill Book Company, New York, 1985).

[23] A. B. Sefkow, R.C. Davidson, P.C. Efthimion, E.P. Gilson, S. S. Yu, P. K. Roy, S. Eylon, F. M. Bieniosek, E. Henestroza, J. W. Kwan, J. E. Coleman, W. L. Waldron, 
W. G. Greenway, D.L. Vanecek, and D. R. Welch, Proceedings of the 2005 Particle Accelerator Conference, 2005, p. 3765, http://ieeexplore.ieee.org/ xpls/abs_all.jsp?arnumber $=1591610$.

[24] W. Rawnsley, AIP Conf. Proc. 546, 547 (2000).

[25] W. Rawnsley (private communication).

[26] F. Bieniosek, D. Baca, P. K. Roy, P. A. Seidl, S. S. Yu, A. W. Molvik, M. K. Covo, and D. Shiraki, Bull. Am. Phys. Soc. 50, 46 (2005), http://meetings.aps.org/ Meeting/DPP05/Event/34615.
[27] P. K. Roy, S.S. Yu, E. Henestroza, A. Anders, F. M. Bieniosek, J. Coleman, S. Eylon, W. G. Greenway, M. Leitner, B.G. Logan and W. L. Waldron, D. R. Welch, C. Thoma, A.B. Sefkow, E.P. Gilson, P.C. Efthimion, and R.C. Davidson, Phys. Rev. Lett. 95, 234801 (2005).

[28] R. C. Davidson and H. Qin, Phys. Rev. ST Accel. Beams 8, 064201 (2005). 